\title{
A comparative study on the kinematic and kinetic analyses of the different levels of axe kick among female taekwondo jins
}

\author{
Changtao Zhou \\ Shandong Sport University, Jinan, 250102, China
}

\begin{abstract}
This study investigated the kinematic and kinetic differences among the different levels of taekwondo axe kick among female taekwondo jins using the Vicon 3D motion analysis system and Kistler force plates. Independent t-tests were used to detect the differences. The high-level group was designated the senior group (SG), and the low-level group was designated the junior group (JG). Kinematics data showed that SG had more pelvis rotation (p $<0.05)$, earlier time of the lowest attract point $(\mathrm{p}<0.05)$, and higher peak value of velocity curve $(\mathrm{p}<0.05)$ than JG. No differences were observed between the two groups in terms of the lowest attack curve. Kinetics data showed that the peak ground reaction forces (GRF) in the vertical direction in SG were higher $(\mathrm{p}<0.05)$ than those of JG; the peak force in SG was also exhibited earlier $(\mathrm{p}<0.05)$ than that in JG. Therefore, differences were detected in the pelvis rotation, peak value in attack velocity, and GRF in the anterior-posterior direction between SG and JG. These factors also increased the attack velocity and range in SG.
\end{abstract}

\section{Introduction}

Taekwondo is a combat sport that uses various kicks, such as crescent, axe, spinning, and back kicks, as the basic offensive technique. Among all these kicks, the axe kick (also called axe kick) is frequently used by most well-known taekwondo jins because of its force, tactical surprise, high striking point, and long-range strike. With the implementation of new taekwondo rules in 2008, actively hitting the head has become a key factor in determining winners and losers in a tournament [1]. The axe kick is primarily used to hit the head; thus, the prevalence rate of its use is increasing[1,5,7]. In the 2008 Beijing Olympics, jins scored a high $13 \%$ rate using the axe kick, and it ranked second among all leg techniques[2]. The current author's previous study showed that the crescent kick is the most extensively used among all leg techniques, but the axe kick is the second most extensively used because of its force, tactical surprise, and high striking point[3].

To date, studies on axe kick biomechanics have considerably concentrated on kinematics. Wang Lei analyzed the axe kick technique using 3D imaging technology and determined that the change in the hip joint of the swing leg is relatively stable. In the process of knee bending, the knee joint movement is more remarkable than those of the toe and ankle joint. The change in the toe and ankle joint is significantly prominent during kicking, when the maximum value is achieved. After executing the axe kick, both toe and ankle joint displacements begin to decrease abruptly. During the entire split process, the change in the joint movement of taekwondo jins is as follows: toe $>$ ankle joint $>$ knee joint $>$ hip joint. Cheng Lei performed a biomechanical analysis of female taekwondo jins during the semifinal and final rounds of the 2006 Taekwondo Championship in China using an SIMI motion 
analysis system. This study obtained the time, angle, velocity, gravitational changes, and other biomechanical parameters of the crescent, front, and axe kicks of female taekwondo jins. The beginning of the knee lifting, kicking, and drawing back leg stages of the swinging leg accounted for $22 \%, 46 \%$, and $32 \%$, respectively. The percentage of time of stage playing kick is higher than those of the crescent and front kicks. In summary, local studies on taekwondo sports biomechanics mainly focused on the technical movements of the kinematics description. These studies included only one research index and mainly underscored angle, speed, body weight, and other parameters. Moreover, strike speed, strike range, and other corresponding indicators were lacking. A comparison of the kinematics of the different levels of taekwondo jins has not been performed. The dynamics of these athletes at different levels is also poorly understood. Therefore, no conclusion has been formed on the foot force characteristics of the axe kick.

The Vicon (Corporation T20-s, Vicon, England) infrared 3D kinematic analysis system is proposed in the current study. By consulting the ground 3D reaction force curve (Kistler, 9287BA, Switzerland), this study synchronously analyzes the kinematics and dynamics of the axe kick and compare the different levels of data of female taekwondo jins. A summary of the technical problems of taekwondo jins and data support and scientific bases for future training is also provided.

\section{Subjects and method}

\subsection{Subjects}

This study recruited 24 female taekwondo jins as subjects. Among these athletes, 11 were national first-grade athletes and were designated as the junior group (JG) in this study. The remaining 13 players were national-level athletes and were designated as the senior group (SG).

No significant differences in height, weight, and age among members of the two groups were noted. Table 1 shows the details of the subjects.

Table 1. Details of the subjects

\begin{tabular}{llllll}
\hline Groups & Number & Height $(\mathrm{cm})$ & Weight $(\mathrm{kg})$ & Age(y) & Training time(y) \\
\hline JG & 11 & $168.23 \pm 3.12$ & $62.14 \pm 5.10$ & $21.22 \pm 2.41$ & $3.1 \pm 0.98$ \\
SG & 13 & $169.45 \pm 4.11$ & $64.26 \pm 6.61$ & $20.10 \pm 1.47$ & $7.4 \pm 3.67$ \\
\hline
\end{tabular}

\subsection{Research method}

\subsubsection{Laboratory setting}

Eight infrared cameras were placed along a laboratory platform to collect the kinematic data of the taekwondo jins. The shooting frequency of the camera was $1,000 \mathrm{~Hz}$. A 3D force platform (Kistler, 9287BA, Switzerland) with a collection frequency of $1,000 \mathrm{~Hz}$ was installed on the platform. A Vicon system was used to synchronize the two instruments. Before the test, 34 reflectors were used to mark the joints of these athletes (full body model of the Vicon system). The diameter of the reflector is 14 $\mathrm{mm}$ (Figure 1). 


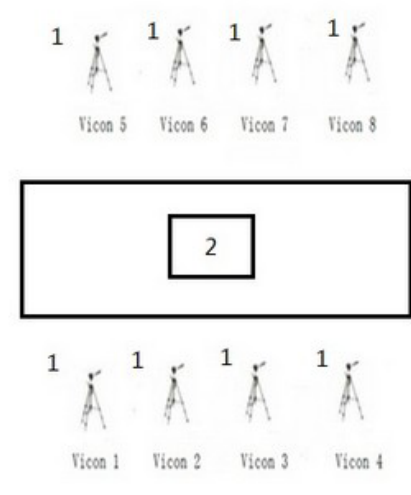

(a). Lab settings

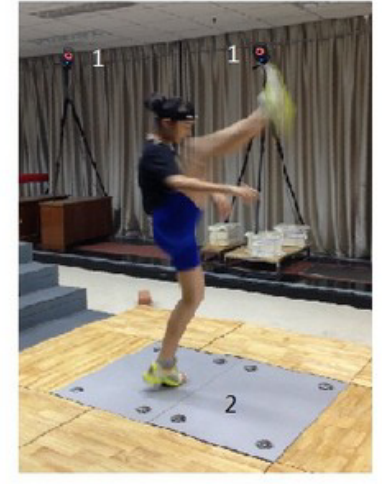

(b). Real Pics

Figure 1. Laboratory setting: 1: Laboratory Setting 1: Vicon infrared cameras 2. Kistlerforce platform.

\subsubsection{Experimental procedures}

All subjects were informed about the details of the trial and signed the agreement. The experiment was conducted at the Laboratory of Sports Biomechanics of the Shandong Sports Institute. Upon arrival at the laboratory, the subjects were informed about the following details: 1) the objectives, significance, procedure, content, method, and notice of the experiment; 2) staff members would record and test their age, height, weight, athletic performance, and so on; 3) they would be asked to practice assessing the test before the actual experiment and to become familiar with the test environment; 4) staff members would place reflective marks on the subjects and debug the test force platform; 5) data would be collected during the experiment; 6) each subject would execute the axe kicks in their usual rhythm; and 7) each subject would repeat the action to extract five valid data; the average value will then be calculated.

\subsubsection{Data processing}

Changes in the angle of the ankle are considerably large. Therefore, the ankle joint of the taekwondo jin is selected as the distal end of the axe kick movement. The distance of the athlete's ankle to the body's center of gravity serves as the strike distance. Similarly, the ankle speed of the swinging leg of the taekwondo jin serves as the strike speed. The angle between the two iliac spine connection and the frontal axis in the horizontal plane is denoted as the rotation angle of the pelvis. The measured plantar reaction force data standardize the center of gravity of the athlete. Each taekwondo jin was required to provide kinematic and kinetic data five times and to be involved in the data analysis thrice.

\subsection{Statistical Analysis}

Data from the two groups were compared using independent t-tests. A type I error rate of below 0.05 indicates a significant difference. 


\section{Research results}

\subsection{Study of the kinematic parameters of the different grades of female taekwondo jins}

\subsubsection{Comparative study of the pelvis rotation}

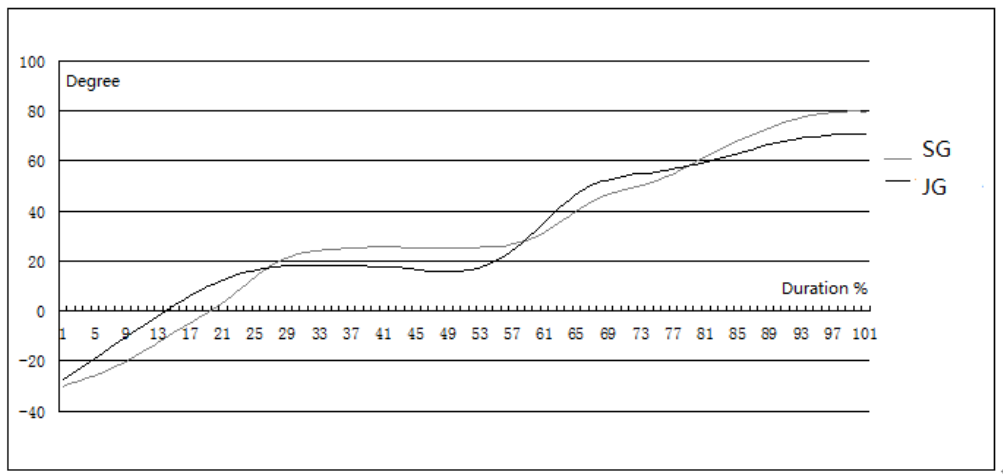

Figure 2. Pelvic rotation angle.

In general, the movement form of the pelvis during the axe kick movement in the horizontal plane is rotary-fixed-rotary.

The pelvic rotation range of $\mathrm{SG}$ was larger in the first rotation stage $\left(\mathrm{SG}=52.65^{\circ}, \mathrm{JG}=46.04^{\circ}, \mathrm{p}\right.$ $=0.006)$ than that of JG. In the fixed phase, the pelvic rotation range of SG was relatively stable (SG $=4.12^{\circ}, \mathrm{JG}=-2.28^{\circ}, \mathrm{p}=0.013$ ). No significant difference was found in the rotation range of the two groups during the second rotation stage $\left(\mathrm{SG}=53.06^{\circ}, \mathrm{JG}=55.17^{\circ}, \mathrm{p}=0.365\right)$ (Figure 2).

\subsubsection{Comparative study of the swing leg range and attack speed}

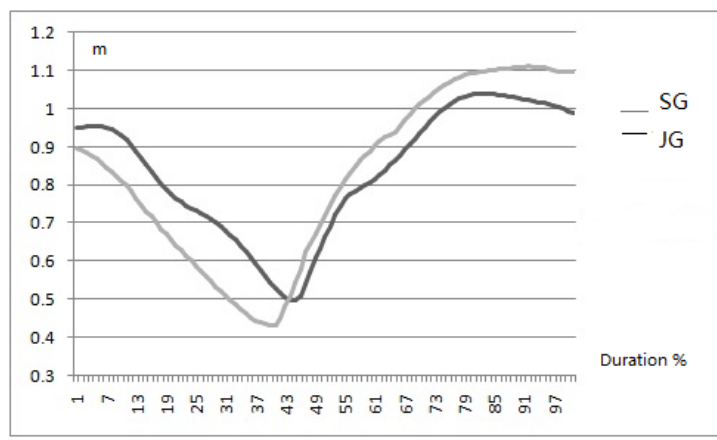

(a) Attack Range

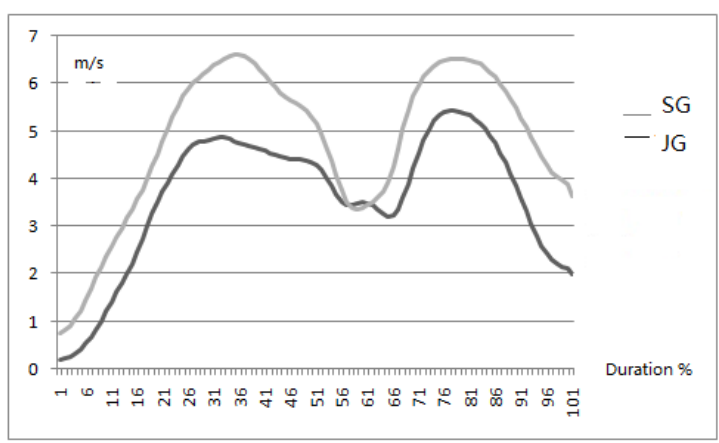

(b) Attack Velocity

Figure 3. Kinematic parameters of the swing leg including (a) swing leg of the strike range and (b) swing leg of the strike speed.

Figure 3 (a) shows that the strike distance curve of the axe kick of the taekwondo jins is generally Vshaped. The lowest point in the SG curve was significantly earlier than that of JG (SG $=41.16 \%, \mathrm{JG}=$ $45.24 \%, \mathrm{p}=0.012)$. The lowest point data of $\mathrm{SG}$ was significantly lower than that of JG $(\mathrm{SG}=0.43 \mathrm{~m}$, $\mathrm{JG}=0.49 \mathrm{~m}, \mathrm{p}=0.021)$. The highest point data of $\mathrm{SG}$ was significantly higher than that of $\mathrm{JG}(\mathrm{SG}=$ $1.11 \mathrm{~m}, \mathrm{JG}=1.03 \mathrm{~m}, \mathrm{p}=0.000$ ). Thus, the swing leg range of JG was considerably small, but the 
swing leg was significantly large upon reaching the lowest point.

Figure 3 (b) shows that the speed curve of the swing leg of SG has two single peaks. Both the SG first peak $(\mathrm{SG}=6.65 \mathrm{~m} / \mathrm{s}, \mathrm{JG}=4.90 \mathrm{~m} / \mathrm{s}, \mathrm{p}=0.000)$ and the second peak $(\mathrm{SG}=6.62 \mathrm{~m} / \mathrm{s}, \mathrm{JG}=5.44$ $\mathrm{m} / \mathrm{s}, \mathrm{p}=0.000$ ) were higher than those of JG. No significant difference was found at the minimum of the swing leg speed curve between the two groups $(\mathrm{SG}=3.36 \mathrm{~m} / \mathrm{s}, \mathrm{JG}=3.19 \mathrm{~m} / \mathrm{s}, \mathrm{p}=0.104)$. SG als o appeared earlier in the valley than $\mathrm{JG}(\mathrm{SG}=58.19 \%, \mathrm{JG}=65.34 \%, \mathrm{p}=0.009)$.

\subsection{Study of the dynamic parameters of the different levels of female taekwondo jins}
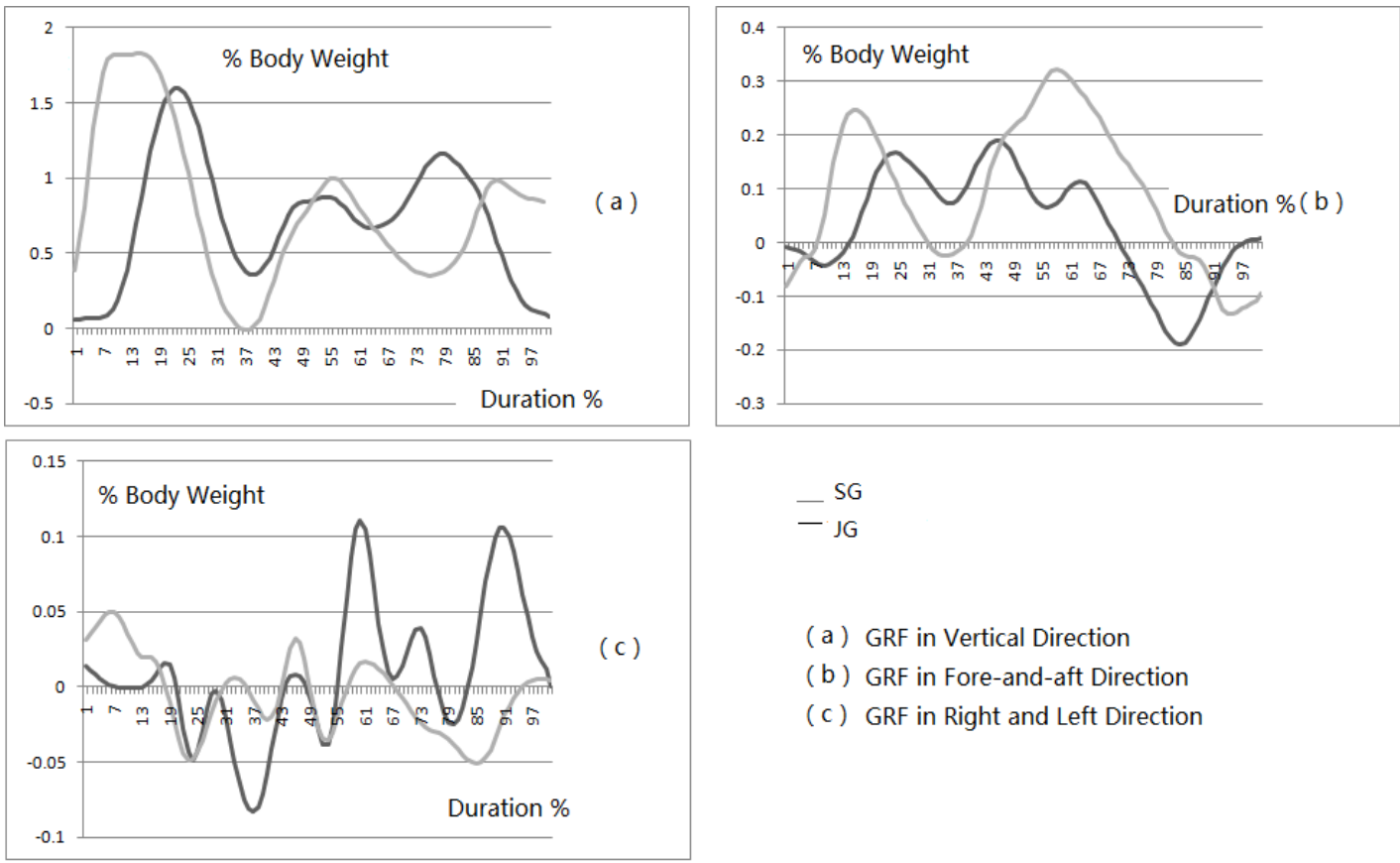

$$
\overline{-}_{\mathrm{JG}}^{\mathrm{SG}}
$$
(a) GRF in Vertical Direction
(b) GRF in Fore-and-aft Direction
(c) GRF in Right and Left Direction

Figure 4. 3D GRF curve

Figure (4a) shows that the ground reaction force (GRF) curve is generally a three-peak curve in the vertical direction. The first peak value of $\mathrm{SG}$ was larger than that of $\mathrm{JG}(\mathrm{SG}=1.77 \mathrm{BW}, \mathrm{JG}=1.60$ $\mathrm{BW}, \mathrm{p}=0.021)$. The first peak of $\mathrm{SG}$ appeared significantly earlier than that of $\mathrm{JG}(\mathrm{SG}=16.54 \%, \mathrm{JG}$ $=20.14 \%, p=0.013)$. No significant differences were noted in the values of the second peak $(\mathrm{SG}=$ $1.01 \mathrm{BW}, \mathrm{JG}=0.93 \mathrm{BW}, \mathrm{p}=0.424)$ and in the appearance time of the second peak $(\mathrm{SG}=56.94 \%, \mathrm{JG}$ $=58.19 \%, \mathrm{p}=0.213)$. The third peak value of $\mathrm{SG}$ was significantly less than that of JG $(\mathrm{SG}=0.99$ $\mathrm{BW}, \mathrm{JG}=1.18 \mathrm{BW}, \mathrm{p}=0.001)$, and the $\mathrm{SG}$ appearance time was significantly later than that of $(\mathrm{SG}=92.74 \%, \mathrm{JG}=80.14 \%, \mathrm{p}=0.000)$.

Figure 4 (b) shows that the GRF direction of the fore-and-aft direction of the taekwondo jins is generally to the front of the body. The peak value of SG was higher than that of JG (SG $=0.32 \mathrm{BW}$, $\mathrm{JG}=0.18 \mathrm{BW}, \mathrm{p}=0.000$ ). Moreover, the $\mathrm{SG}$ members received a considerably high impulse (force and time integral) to the front of the body.

Figure 4 (c) shows that the GRF curves of the right and left direction fluctuate up and down around the axis, but the volatility is large in this parameter. GRF to the left direction (negative value) of $\mathrm{SG}$ was smaller than that of $\mathrm{JG}(\mathrm{SG}=-0.05 \mathrm{BW}, \mathrm{JG}=-0.08 \mathrm{BW}, \mathrm{p}=0.001)$. By contrast, GRF to the right direction (positive value) of $\mathrm{SG}$ was smaller than that of $\mathrm{JG}(\mathrm{SG}=-0.03 \mathrm{BW}, \mathrm{JG}=-0.11 \mathrm{BW}$, $\mathrm{p}=0.000)$. 


\section{Discussion}

Analysis of the blow range curve indicates that the value of SG in the front section of the curve is less than that of JG. However, in the back section, the value of SG is lower than that of JG, and the appearance time of the lowest point in SG is earlier than that in JG. The front section values mainly correspond to the initial ankle joint action upon reaching the top position, i.e., the preliminary swing. The values of the back end mainly correspond to the completion of the swing leg action. From the curve, the distance between the ankle joint and the center of gravity of the body in SG is smaller in the preliminary swing than that in JG. This result may be caused by high-level athletes completing preliminary swinging with a considerably large flexion angle of the lower extremity joint $[4,9,11]$. A large flexion angle can shorten the swing circumference of the swinging leg, thus reducing the swing movement time. At the back section of the curve, the strike range of SG is larger than that of JG. This result may be caused by high-level athletes completing a axe kick with a small flexion angle of the lower extremity joint $[4,13]$. Analysis of the speed curve shows that the values in the front and back of the curve of SG are less than those of JG. The appearance time of the lowest point of SG is earlier than that of JG. Speed curve shows that regardless of the preliminary swing or completion of the action stage, the SG ankle joint velocity index is greater than that of JG. The completion time of SG is lower than that of JG. SG athletes increase the flexion angle of their lower extremities and complete the swing movement rapidly. Moreover, the increased range and speed of the kick strengthens the effect.

Studies on the GRF curve in the axe kicks of taekwondo jins have limited involvement in the current study. Based on the analysis of the vertical direction of the curve, the value of the SG first peak is higher and the appearance time is earlier than those of JG. No difference exists between SG and JG in the second peak in terms of value and appearance time. The value of the SG third peak is lower and the appearance time is later than those of JG. Analysis of the peak value function indicates that the first peak is the GRF support during the swing process. The second and third peaks are supported by GRF in the splitting of the swing leg process. Figure XX shows that the SG first peak value is larger than that of JG, thus indicating that the GRF support is larger. The shadow area of the SG first peak (integral of the supporting force and supporting time) is greater than that of JG. This result indicates that the GRF impulse of SG is greater than that of JG. Therefore, the SG athletes move their center of gravity in a considerably large range during the swing movement, thus increasing the swing leg range. Increasing the gravitational potential energy of the starting position can also augment the kicking effect. The SG third peak value may be caused by the high acceleration of the swing leg. During the splitting process, other parts of the athletes' body associated with the swinging leg form a rigid system. The vertically downward acceleration of the swing leg can cause other body parts to move up and thus reduce GRF.

Analysis of the GRF curve of the fore-and-aft direction indicates that the GRF direction of the fore-and-aft direction of the taekwondo jins is generally to the front of the body. The SG peak value and impulse (force and time integral) are higher than those of JG. The considerably large GRF peak value and impulse can increase the forward movement amplitude of the SG athletes, thus effectively increasing their attack range. Analysis of the GRF curve of the left-to-right direction shows that the curve is promiscuous, as it generally fluctuates up and down around the axis. The SG GRF peak value of the left-to-right direction (negative value) is smaller than that of JG. As the opponent of a taekwondo jin is expected to be in front, the former does not need to exert the left-to-right direction force to achieve the objective of an accurate attack. Therefore, the component GRF force in the leftto-right direction plays a role only in maintaining balance in this study. The SG fluctuation range is considerably small, indicating that the taekwondo jins in this group have strong ability to balance their body. Moreover, the SG athletes no longer need the component left-to-right direction force to adjust balance. Therefore, the peak force is reduced effectively.

Analysis of the pelvic rotation range in the horizontal plane indicates that the SG rotation range is larger in the front of the curve than that of JG group. The SG pelvis rotation in the fixed stage is lower than that of JG. Moreover, no difference exists between the rotation ranges of the two groups in the back section of the curve. Values of the fore part correspond to the preliminary swing from the initial 
stage of the swinging leg of the top-most ankle position. Middle values correspond to the athlete completing the kicking movement after the preliminary swing. Values of the back part correspond to the stage after the kicking action, i.e., from bringing it back to letting the swinging leg touch the ground. Based on the analysis of the curve, the SG pelvic rotation range is relatively large, and thus the kicking distance of the SG taekwondo jins in the horizontal direction is considerably large. Consequently, the trunk rotation range is increased, and the attacking range of the opponent is relatively small $[13,15]$. As the pelvic rotation range of the $S G$ athletes is small, the athletes can fix their pelvis better in the process of completing the kick. Consequently, the stability of the body of the SG taekwondo jins is higher than that of the JG jins.

\section{Conclusion}

The rotation range of the SG taekwondo jins during the preliminary swing is larger than that of the JG taekwondo jins. The pelvis is stable, and the stability of the body is improved. The kicking movement is completed rapidly by increasing the flexion angle of the swing leg, the first peak value of the vertical direction GRF and appearance time, and the peak value and impulse of the horizontal direction GRF. The axe kick increases the range and speed of attack, thus strengthening the effect of such an attack. Therefore, the training of low-level athletes should involve the enhancement of body stability, improvement of the flexion and extension angles of the swing leg, enhancement of the peak value of the vertical direction GRF and time, and improvement of the impulse and peak value of the horizontal direction GRF.

\section{References}

1. Fei Ying, Song Hua. The analysis and research of the competition rules of the taekwondo in 2008[J].Martial arts and professional boxing teaching,2009 (9):86.

2. Yang Zhijun. The study of the technical and tactical research of the current situation under the new rules of the excellent taekwondo athletes in our country[J].Sports science and technology in China,2010,46(6): :79-81.

3. Zhou Changtao, Yu Daifeng. The biomechanical characteristics analysis of the Roundhouse Kick of the male taekwondo athletes[J],Journal of Shandong Sports Institute,2010,26( 9) : 58-63.

4. Wang Lei. The biomechanical characteristics analysis of Next Splitting Technique of the excellent female taekwondo athletes at ShanDong,2012, the master's degree thesis of Shandong Sports Institute.

5. Cheng Lei. The biomechanical characteristics analysis of the kick, front kick, chop action of the female taekwondo athletes,2005, the master's degree of Zhejiang Normal University.

6. LuWenchao. The application and the development characteristics of the taekwondo competition tips [J].Sports science and technology,1999,22(1):94-97.

7. Liu Baocheng, Zhao Qiurong, Lu Fan. The Research Report of Chinese Taekwondo Championships 1996 "Wanji Cup" [J].Journal of Xian Sports Institute,1996,14(1): 48-51.

8. Scott L. Delp, William E. Hess, David S ect. Variation of rotation moment arms with hipflexion[J]. Journal of Biomechanics. 32 (1999) 493-501.

9. Liu Baocheng, Zhao Qiurong, Lu fan etc.The technology analysis of 1997 "Kang Cup" National Taekwondo Championship [J]. Journal of Xian Sports Institute.1998,15(2):37-41.

10. Pieter W, Zemper E.D. Time-loss Injuries in Junior Olympic Taekwondo Athletes[J]. Occupational Health and Industrial Medicine Volume: 36, Issue;6,1997,pp.290.

11. Zhu Hui, Xue Xinxuan. The comparative analysis of the application of leg techniques of Chinese and foreign male's taekwondo athletes[J].Journal of Wuhan Sports Institute.2002,36(6):149-150.

12. Zhang Qingfeng, Meng Zhaofeng. Factors affecting the speed of action in Taekwondo[J]. Journal of Zhejiang Normal University(Natural Science Edition).2004,27(3):299-302.

13. Shang Hua, Wang Guanghua, Tu jun. The injury of juvenile Taekwondo, Sanda and boxing[J].Journal of Wuhan Sports Institute. 2000,34:91-92. 
14. Li Zhigan, Chen Runlin. Research on the characteristics of sports injuries of the Taekwondo Athletes in Guangdong Province[J].Journal of Guangzhou Sports Institute.2000,20(2):56-60.

15. Zhu Lihua, Liu Bai, Zhao Xin etc. Investigation and analysis of sports injuries of Chinese excellent taekwondo athletes[J].Journal of Beijing Sports Institute.2001,24(2):76-78, 182. 